\title{
La retribución sociodeportiva del club atlético Huila al departamento del Huila y al municipio de Neiva
}

\section{Resumen}

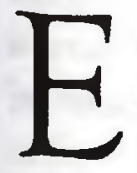
sta es una investigación cualitativa inspirada en el paradigma interpretativo, por lo tanto la relación entre la teoría y la investigación es interactiva, se trata de indagar acerca de las oportunidades que Club Atlético Huila da a la comunidad huilense como contraprestación por el apoyo económico de los gobiernos departamental y municipal para su funcionamiento. Se presenta una postura acerca de la relevancia que ha adquirido el deporte en general y el fútbol en particular el cual se ha insertando aceleradamente en la vida cotidiana del hombre.

Se reseña la evolución de la figura del Club deportivo profesional de fútbol y el carácter empresarial monopólico que ha adquirido modelo que también configura el Atlético Huila, en éste se plantea la influencia del Club en la región.

\section{Introducción}

Los estudios históricos normalmente se han impuesto dos fuertes límites; el primero, más o menos compartido con las demás disciplinas de
Jaime Monje Mahecha Catedrático de la Facultad de Educación

Universidad Surcolombiana jaimemoma@hotmail.com

Bajo la premisa anterior, pretender adoptar el deporte como tema de investigación se descalificaría por realizarlo sobre algo "no serio" como lo es el deporte (que puede tener como uno de sus sinónimos al juego, antítesis de "lo serio"). En el citado libro, Norbert Elias, comenta la discusión con Eric Dunning, acerca, de si el deporte, y el fútbol en particular, sería considerado por las autoridades un tema respetable de investigación en las ciencias sociales y, sobre todo, para una tesis de maestría. Más adelante dice "Los estudios del deporte que no son estudios de la sociedad, son estudios fuera de contexto". Esta afirmación de Elías, pone de manifiesto la necesidad de articular nexos, que el deporte va tejiendo con otros aspectos inherentes al desarrollo histórico de los pueblos. El profesor Macías Cervantes al respecto afirma "El fenómeno del deporte encierra una historia tal vez propia pero no aislada de la sociedad que los produce. Ver a un fenómeno deportivo aislado en su propio espacio, en su excluyente 
temporalidad, limitado a la cancha, la pista, o la piscina, a sus propios protagonistas inmediatos es perder toda posibilidad de riquezạ de análisis histórico". El deporte y su infraestructura son proporcionales a la sociedad en que se desarrolla su práctica. (Macías Cervantes, 2006).

Las prácticas deportivas deben verse enlazadas plenamente a las posibilidades materiales para su desenvolvimiento, condicionadas por las consideraciones morales de la sociedad que las aloja, impulsadas por los intereses sociales del entorno, encausadas por los intereses económicos de quien las patrocina.

Antonio Rovira i Virgil ya desde 1926 anotaba: ¿Por qué no he de tratar en esta revista y con mi firma el problema del futbol en Cataluña? Este problema no es hoy simplemente deportivo. Hace tiempo que ha pasado a la categoría de problema social y espiritual.

Un juego que reúne a veinte o treinta mil espectadores en el estadio y a cien mil manifestantes en la calle; que engulle columnas y páginas enteras de la prensa diaria; que es el principal elemento sustentador de una numerosa prensa deportiva; que fleta navíos, organiza trenes especiales y hace que centenares de automóviles corran raudos por las carreteras; que abarrota los hoteles, las fondas, los hostales y las casas de huéspedes en los días de partidos scnsacionales o decisivos; que absorbe el interés de la gente y es el tema predominante en las conversaciones durante semanas y meses... este fenómeno entra de lleno en la categoría de fenómeno social." . (Santacana Torres, 2006) Pareciera que un camino hace tanto tiempo empezado a andar ya nos tendria en un grado avanzado, pero iniciaba el siglo XXI y desde la misma España, tierra de Rovira, se escribía respecto a la historiografía deportiva:
Predominan entre los libros dedicados a la historia del futbol, los anecdotarios, las crónicas de clubes y las historias de acontecimientos. En este sentido, quizá el grupo más numeroso sea el de las historias de los clubes modestos, que son tratados con una erudita meticulosidad, pero un tanto alejados de los estándares académicos y realizados por periodistas, eruditos locales y aficionados al futbol y especialmente a su equipo (Capistegui, 2001)

Los Clubes de fútbol están íntimamente ligados con la ciudad que los acoge, se podrian citar nexos en todos los continentes y países y su reciproca influencia. En el texto La construcción de representaciones sociales a través del discurso textual. El club de fútbol del Real Zaragoza (España). Cantarero Abad comentando acerca de las políticas municipales y autonómicas en torno al Real Zaragoza afirma.

Qué duda cabe de que las politicas deportivas son fundamentales en el devenir del deporte. Básicamente, lo que a los clubes profesionales les interesa de las administraciones públicas es conseguir dinero. En Aragón existe la posibilidad de solicitarlo al ayuntamiento y al Gobierno de Aragón. El primero tiene responsabilidades locales; el segundo, regionales.

El Ayuntamiento de Zaragoza, a través de su programa Zaragoza Deporte Municipal, gestiona esta actividad. Las actuaciones políticas pueden ser diversas y abarcan un continuo cuyos polos los ocupan, en un extremo, el potenciar el deporte de élite (alta competición); en el otro, el de masas ("para todos"). Por un lado, se trata de favorecer a los equipos locales de alcance nacional e internacional alegando que así se contribuye a promover la ciudad y, en consecuencia, se entiende como una inversión politica y económica que puede ser rentable. Por 
otro, se busca potenciar el "deporte para todos", es decir, generalizar la actividad fisica entre los ciudadanos con el fin de mejorar su condición física, psicológica y social. (Cantero Abad, 2007) La comunidad y la prensa local justifica la aportación presupuestaria en términos de "beneficio para la ciudad", basándose en datos que señalan la relevancia del equipo fuera de sus fronteras: "seiscientos periodistas de diferentes países cubrieron el partido (la final de la Copa del Rey) y emitieron setenta y cinco televisiones internacionales" (Heraldo de Aragón, 03/03/ 2004). El lector da por sentado que el citado recorte presupuestario no debe afectar al equipo: ¿cómo no va a financiarse al Real Zaragoza que tantas miradas congrega? Si además lo sentimos como "nuestro", como un referente con el cual identificarnos, las opiniones a favor de la "donación pública" están servidas. En cualquier caso, se deja constancia del carácter simbólico del equipo de fútbol, gracias al cual representa a la ciudad que lo acoge, y afirma que el devenir de ambos corre de la mano. Esta asociación. ciudad-equipo de fútbol-permite recrear el sentimiento de pertenencia en la ciudadanía de Zaragoza.

Vemos como el deporte, pero especialmente del fútbol se ha insertado paulatinamente en la vida cotidiana del hombre, como espectáculo deportivo se ha constituido en proveedor lúdico, al que se le invierte tiempo y recursos, con una dinámica arrolladora ha creado empleos específicos que en la historia de la humanidad no se habian visto nunca, como cronistas deportivos, "advertido por Antonio Rovira ya en 1926", narradores de radio y televisión, promotores, entrenadores, detectores de talentos, rehabilitadores, directivos y por supuesto futbolistas que se dedican exclusivamente a esta actividad, deporte que ha ensanchado la nomenclatura de héroes, ha creado identidades mediante simbolos representativos, como banderas u otros objetos.

Es tal la importancia de este fenómeno deportivo que en un país como México, (con la cuarta Liga más lucrativa del mundo) los clubes de fútbol son financiados por los gobiernos de los Estados. En San Luis Potosi el gobierno del Estado gastó el $70 \%$ de su presupuesto para deporte en comprar boletos para el equipo de futbol San Luis de forma que los estadios no estuvieran vacios. Jaguares de Chiapas, consume el $80 \%$ de los recursos destinados al deporte en este Estado de la República mexicana. Lo mismo puede afirmarse de Michoacán donde la remodelación del estadio fue financiada por el gobierno del Estado.

\section{Club Atlético Huila}

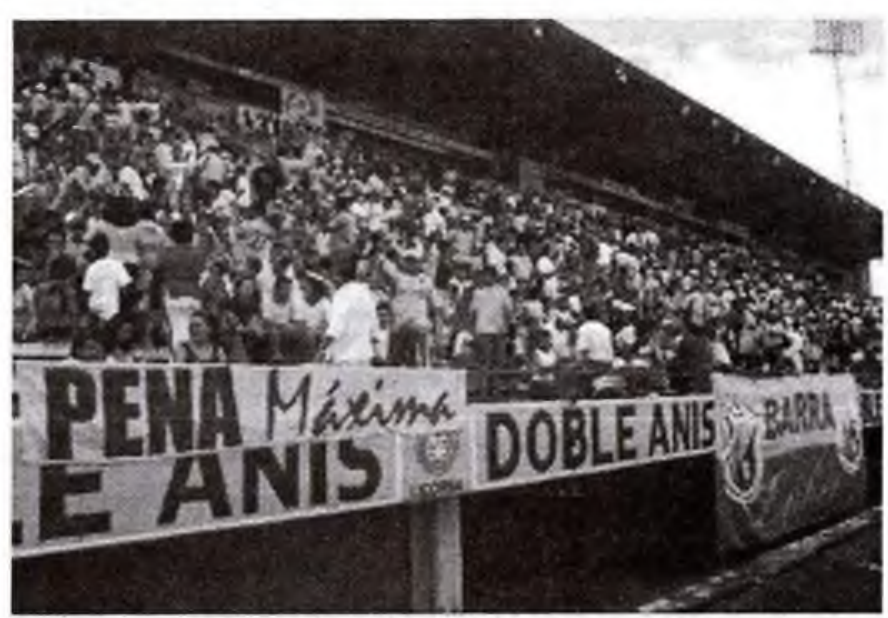

La ciudad de Neiva no es ajena a este fenómeno sociológico, alberga al Atlético Huila, Club deportivo que promueve la práctica del fútbol profesional, el cual "admite como competidores a personas naturales bajo remuneración, de conformidad con las normas de la FIFA". Hizo su debut en la categoría Primera B de la División Mayor del fútbol colombiano en 1991, logró el cupo en la Primera A al ganar la Copa Concasa de 1992, el anhelo de todos los huilenses de ver un representativo de su departamento 
disputando el torneo de la Primera A del fútbol profesional colombiano se hizo realidad desde la temporada de 1993, su participación en esta categoría solamente se interrumpió al final de la temporada del 95 cuando perdió la casilla durante los años 96 y 97 recuperándola nuevamente hasta nuestros días.

Durante todos estos años el club ha tenido dificultades de indole económica y los gobiernos departamental y municipal siempre lo han auxiliado. Como referencia podemos decir que en 1990 cuando se constituyó el Club Deportivo Atlético Huila para participar en el campeonato de Primera B los aportes de los gobiernos departamental y municipal fueron de 10 y 40 millones respectivamente.

El periodista Luis Guillermo Ordóñez comenta "El Huila, un representante de los equipos llamados 'chicos', tiene un presupuesto modesto y poca historia en la primera división, pues apenas ha jugado una final, la que perdió en 2007 ante Nacional." (Ordoñez, 2009) Los $\$ 4.500$ millones recaudados en el 2009 son un presupuesto reducido teniendo en cuenta las necesidades de un Club profesional de Fútbol, hay que considerar las asimetrias en las economias regionales, mientras que un equipo como Atlético Nacional, en un partido de campeonato regular puede recaudar en la ciudad de Medellín \$ 800 millones, en Neiva una excepcional taquilla en una instancia definitiva del campeonato, por ejemplo disputando finales puede llegar a $\$ 100$ millones. Afortunadamente esta diferencia en ingresos no es proporcional en el desempeño deportivo y varias veces hemos visto que equipos con nóminas costosas son superados por equipos integrados por jugadores a préstamo de divisiones inferiores.

Debido a los altos costos de operación, el modelo de desarrollo del club deportivo basado en el pago de una membrecía por parte de sus socios, solo es funcional para una sociedad que cuenta con sus necesidades básicas cubiertas y donde hay una gran clase media como en muchos países europeos.

Debido a las condiciones socioeconómicas mundiales actuales este modelo ha sufrido importantes cambios durante los últimos años. Antes, tradicionalmente los clubes se componían de miembros con igual peso en las decisiones a través de una asamblea y una mesa directiva. Actualmente, muchos de estos, se transformaron en empresas las cuales tienen un propietario o accionista mayoritario donde el peso de quien ha invertido más es lo que determina la toma de decisiones.

Los orgullosos ingleses han tenido que ver impasibles como clubes emblemáticos como el Manchester City, Club fundado en 1884 ha sido controlado desde el 1 de septiembre de 2008 por Abu Dhabi United Group for Development and Investment, un grupo inversor de los Emiratos Árabes con Sulaiman Al-Fahim como parte visible del grupo, por un monto cercano a los 250 millones de euros, relevando así al hasta ese momento dueño de la entidad, Thaksin Shinawatra, o el Chelsea fundado en 1905 , adquirido en el año 2003 por el joven magnate del petróleo, el billonario_ruso Román Abramóvich. Adquisiciones que se asimilan más a un capricho personal o adquisición de estatus que a una inversión en busca de rentabilidad económica.

En el departamento del Huila, y en la ciudad de Neiva, la aplicación del modelo del club deportivo tampoco es viable, hay una gran concentración de riqueza en pocas manos y el interés principal de la comunidad gira en torno a la sobrevivencia. El escaso tiempo libre y los salarios bajos hacen imposible un desarrollo en 
torno a esta figura. Por esto al igual que los casos citados anteriormente, el Atlético Huila tiene un accionista cuyo peso específico no deja margen de dudas de quien toma las decisiones.

Dado su carácter de empresa privada, el Atlético Huila busca ingresos de muchas fuentes, entre otras: el aporte de sus socios, publicidad en la camiseta, alquiler de vallas publicitarias, compra y venta de jugadores, entradas a los partidos etc. Sin embargo al igual que el Real Zaragoza la ayuda Estatal no solo no es desestimada sino que hace parte de su presupuesto.

\section{Financiación}

El Atlético Huila sabe que como institución es fuente de identificación del huilense y a partir de esta condición genera gratificación a la comunidad, por lo tanto espera que las administraciones públicas lo respalden económicamente. Por supuesto los gobiernos también lo saben y hacen su respectivo aporte. Lo deseable es que dicho aporte fuera directo, abierto, no enmascarado, sobre todo porque pautar con el Atlético Huila para la gobernación o alcaldia es redituable en términos de publicidad ya que el Departamento y la ciudad son mencionados casi a diario por los diversos medios de comunicación locales, nacionales e internacionales, además un club deportivo de estas características es una institución de alto valor y significado para una región.

En respuesta al derecho de petición GH017041 del 14 de abril de 2009, formulado por el grupo de investigación Identidad, con relación a los aportes realizados por el departamento del Huila al Club deportivo Atlético Huila, para la vigencia fiscal de 2001 el señor Secretario de Hacienda departamental Carlos Andrés Cante Puentes hizo las siguientes precisiones:

\begin{tabular}{|l|l|c|}
\hline DEPENDENCIA & \multicolumn{1}{|c|}{ CONCEPTO } & VALOR \\
\hline $\begin{array}{l}\text { Secretaría de } \\
\text { Hacienda }\end{array}$ & $\begin{array}{l}\text { Contrato para el fomento de la formación } \\
\text { física deportiva }\end{array}$ & 166.000 .000 .00 \\
\hline $\begin{array}{l}\text { Secretaría de } \\
\text { Salud }\end{array}$ & $\begin{array}{l}\text { Campaña publicitaría sobre promoción de } \\
\text { la salud y prevención de enfermedades }\end{array}$ & 32.000 .000 .00 \\
\hline \multicolumn{1}{|c|}{ Total } & & 198.000 .000 .00 \\
\hline
\end{tabular}

Observamos como la legitima contribución que puede hacer el gobierno departamental a un Club deportivo como el Atlético Huila, para que nos represente dignamente en el marco del campeonato organizado por la división profesional de la Federación Colombiana de Fútbol, en ese entonces denominada Copa Mustang, es mimetizada por unos contratos otorgados por las Secretarías de Hacienda y Salud al club, para los cuales el Atlético Huila no está habilitado, ni es función de la Secretaría de Hacienda departamental fomentar la formación física.

En la vigencia fiscal de 2006, las citadas secretarías acuden al Atlético Huila para: 


\begin{tabular}{|c|c|c|}
\hline DEPENDENCIA & CONCEPTO & VALOR \\
\hline $\begin{array}{c}\text { Secretaría de } \\
\text { Salud }\end{array}$ & $\begin{array}{l}\text { Contrato N. } 769 / 06 \text { prestación de } \\
\text { servicios para el desarrollo de jornadas } \\
\text { promocionales y aplicación de encuestas } \\
\text { conocimientos aptitudes y practicas } \\
\text { relacionadas con factores de riesgo, } \\
\text { incrementar niveles de actividad física en } \\
\text { desarrollo de la estrategia información } \\
\text { adecuación en programas de salud sexual } \\
\text { y reproductiva }\end{array}$ & 75.000 .000 .00 \\
\hline $\begin{array}{l}\text { Secretaría de } \\
\text { Salud }\end{array}$ & $\begin{array}{l}\text { Convenio de cooperación n. } 51 / 06 \text { del } \\
\text { contrato de prestación de servicios para la } \\
\text { cooperación de carácter deportivo y sin } \\
\text { ánimo de lucro que coopere en la } \\
\text { implementación y ejecución de las } \\
\text { estrategias de los programas de salud } \\
\text { sexual y reproductiva, salud mental y } \\
\text { promoción de estilos de vida saludable } \\
\text { para prevención y control de } \\
\text { enfermedades crónicas en el en } \\
\text { departamento del Huila }\end{array}$ & 75.000 .000 .00 \\
\hline \multicolumn{2}{|r|}{ Total } & 150.000 .000 .00 \\
\hline
\end{tabular}

Hay instituciones de carácter académico encargadas de diseñar programas de salud sexual y reproductiva, de la promoción de estilos de vida saludable para la prevención y control de enfermedades crónicas en el departamento del Huila, sin embargo además de velar por el rendimiento deportivo del conjunto opita, al Atlético se le suman estas otras tareas.

Con este tipo de contratos se expone el departamento y el Club a una investigación con sus respectivas consecuencias civiles y penales porque el cumplimiento de los mismos es muy dudoso, ya que el Club no está capacitado para ejercer las funciones asignadas en ellos ni hay evidencias de su cumplimiento.

La Gobernación debe ser explícito y dar los recursos que estime convenientes al Club con la expresa intención de apoyar su funcionamiento para que mejore sus resultados deportivos y/o la intención que requieran, pero no hacer este tipo de contrato que no son creíbles, situación que corrigió en la vigencia del 2009 otorgando al Club Atlético Huila $\$ 1.500$ millones para su funcionamiento a través del INDERHUILA la Institución destinada a manejar el deporte en el Huila.

Con relación al municipio de Neiva, de acuerdo con el Señor Andrés Cardozo a través del Acuerdo Municipal 012 de 2003, se determinó en su artículo primero otorgar el beneficio de exoneración para:

- Los Clubes Deportivos de Profesionales en sus diferentes disciplinas, que tengan su sede en la ciudad de Neiva, en un por ciento (100\%). Léase Atlético Huila. 
Dicha exoneración en términos económicos significa que el Atlético Huila se ha beneficiado en aproximadamente $\$ 30.000 .000$ anuales, contados a partir del 2003 fecha de aprobación del referido Acuerdo Municipal. Los aportes dados por el Municipio en los años siguientes no nos fueron suministrados

Está claro, que con taquillas no se sostiene un club, aún con una nómina contenida como la del Atlético Huila. Aunque cuantiosos, resultan insuficientes los recursos que destinan los gobiernos departamental y municipal para su sostenimiento, debido a esto acude a patrocinios

\section{Retribución a la Comunidad}

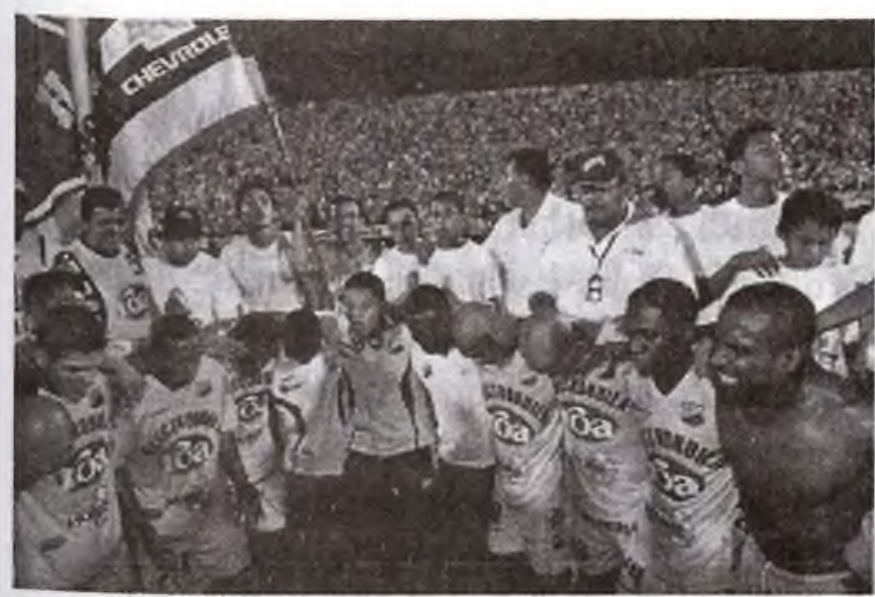

Ante estas inquietudes surgen unas preguntas, además de ser fuente de identificación del huilense, de generar gratificación en la comunidad y promover la ciudad y el departamento ¿Qué otros beneficios aporta el Club Atlético Huila, a la región? ¿Los aportes al Club por parte de los gobiernos departamental y/o municipal van en detrimento de la función social de promover el "deporte para todos"?

Se espera que la dinámica operativa del Atlético Huila brinde oportunidades de diversa indole para la región, como: la oferta laboral a profesionales, médicos, fisioterapeutas, técnicos privados, por esta vía consiguió $\$ 3.000$ millones, entre los patrocinadores más representativos están: Electrohuila, Molino Roa, Coomotor, Aires, Cafesalud, Emgesa, La Nación. El patrocinador privado que más aporta es Colpatria con $\$ 1.000$ millones.

La percepción de la comunidad es que dado su carácter privado, los clubes deportivos no tienen que rendir cuentas, por lo tanto quedan a merced de la lógica del capital. Se piensa que están más preocupados por cuanto van a recibir de ingresos por patrocinios que por masificar la práctica deportiva.

deportivos, Licenciados en Educación Física, administradores deportivos; además a través del montaje quincenal o semanal del espectáculo deportivo reactive la economía informal, desde vendedores ambulantes hasta cuidadores de carros.

\section{NuestrosJugadores}

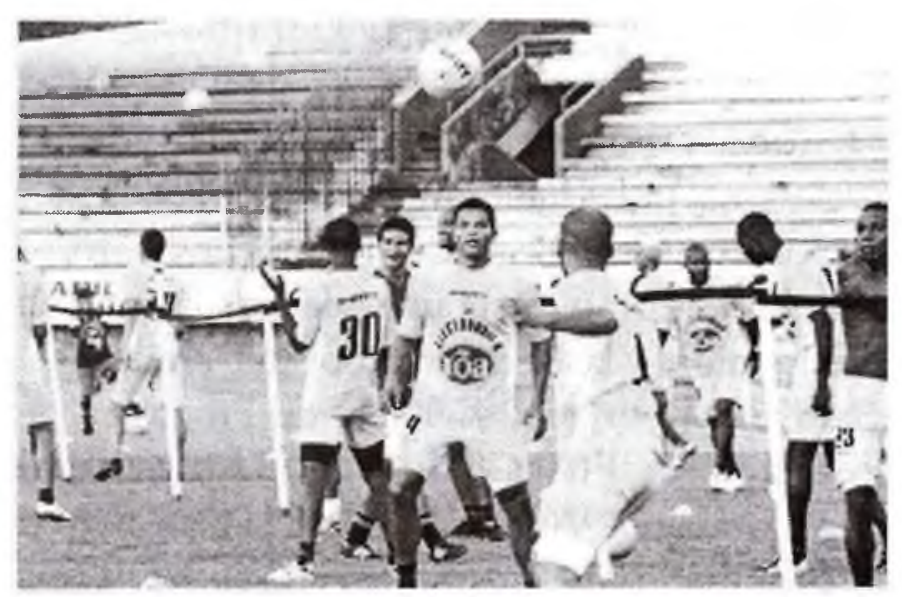

Los activos más visibles y rentables en un Club profesional de fútbol son los jugadores, quienes a partir de su rendimiento se vuelven ídolos de la afición, los cuales garantizan en alguna medida más asistencia a los estadios que redunda a su vez en apoyo económico. Para la afición huilense 
la posibilidad de formar y darles la oportunidad de participar a jugadores oriundos del departamento es uno de los anhelós que aún no se ha cumplido, el Atlético Huila acude con frecuencia a jugadores en préstamo de equipos denominados "grandes", esto tiene una doble connotación, de un lado se le niega la oportunidad a un jugador oriundo de nuestra región y al mismo Club la posibilidad de aumentar la cantera de jugadores para acrecentar su patrimonio, por otro, como ha sucedido año tras año maduran y hacen visibles a jugadores anónimos en sus clubes de origen, que rápidamente se valoran y son solicitados nuevamente para hacerlos parte de su nómina o venderlos buen precio.

Indagando sobre las posibles causas del escaso número de jugadores juveniles de fútbol del Huila que logran llegar y consolidarse en el fútbol profesional colombiano, Barreto encontró. (Diego Armando, 2008). Expertos en la materia que tienen un amplio recorrido con el fútbol en el departamento del Huila entre ellos se encuentran; el Gerente Deportivo del Club Deportivo Atlético Huila Gerardo Gómez Álvarez, el anterior Director de Divisiones Menores y hoy director técnico del equipo profesional Luís Guillermo Berrio, los Periodistas Deportivos José Diego Cardoza y Faiver Hoyos Hernández como integrantes del grupo focal, el presidente de la liga de fútbol del Huila Yael Garaviño Rodríguez y el Jefe de Programa de Educación Fisica de la Universidad Surcolombiana Virgilio Puerto Polanco fueron abordados para consultarles sobre estas inquietudes.

El gerente deportivo del Club Deportivo Atlético Huila, en su intervención reconoce ampliamente que sin duda alguna el fútbol huilense atraviesa desde ya hace varios años una serie de problemas que están incidiendo directamente en la poca producción de jugadores de primer nivel que logren llegar y consolidarse en el fútbol profesional colombiano, y dice "este problema afecta claramente al equipo profesional de esta institución ya que nos vemos en la penosa necesidad de contratar a jugadores profesionales de otras partes del pais y hasta del exterior por grandes cantidades de dinero pudiendo pagar menos si tal vez acá en el departamento hubieran bueno jugadores"Y menciona varios ejemplos, en donde hace referencia a jugadores que en su momento tenían unas magnificas condiciones para jugar al fútbol pero que por falta de algunos aspectos inherentes a la falta de rigor profesional, no lograron llegar a la primera división y mucho menos consolidarse.

Los periodistas deportivos Faiver Hoyos Hernández Presidente de la Asociación Colombiana de Periodistas Deportivos "ACORD HUILA " y José Diego Cardoza Sánchez, Periodista deportivo de Caracol coinciden en sus apreciaciones con el gerente deportivo del Club Deportivo Atlético Huila de que el número de jugadores que logran llegar y consolidarse en el fútbol profesional colombiano es muy exiguo. Además señalan con absoluta claridad que "es la principal razón para que el equipo profesional de nuestra tierra tenga la necesidad de acordar con jugadores de otras partes de pais y del exterior el préstamo de sus servicios a fin de conformar un equipo competitivo y de primer nivel que represente dignamente los colores del departamento, porque en el departamento no contamos con esa clase de jugadores". Hay consenso en que algo está fallando en el proceso de formación de jugadores para que el Huila pueda tener un jugador en el fútbol profesional.

En conversaciones con el técnico del Atlético Huila, Luís Guillermo Berrio Gómez, quien fue Director de Divisiones Menores del Club 
Deportivo Atlético Huila reconoció que los jugadores que el departamento del Huila aporta al fútbol profesional colombiano y en especial al equipo profesional de esta institución es muy escaso, hecho que sucede por diferentes causas bien conocidas por todo el personal que está vinculado al fútbol huilense. Y señala. "En el largo tiempo que llevo vinculado a la institución y al fútbol huilense tanto como jugador y ahora como entrenador es suficiente para detectar claramente que los jugadores que están en categorias próximas al equipo profesional no logran ascender por muchos factores en los cuales estamos fallando todos los que estamos involucrados en este tema, y por ende la presencia de jugadores de la tierra en el equipo profesional de la institución es muy escasa, y si no llegan acá al fútbol profesional dificilmente podrian llegar en otro departamento". Sin embargo no precisa una causa, es muy genérico

El Presidente de la Liga de Fútbol del Huila Yael Garaviño Rodríguez, Presidente Liga de Futbol del Huila, Rector Colegio Cooperativo Utrahuilca opinó sobre el tema y remarcó, "los jugadores que logran llegar al fútbol profesional es minimo porque no tienen las herramientas necesarias ni suficientes para poder lograrlo, asi de esta forma son mucho los jugadores con excepcionales condiciones para jugar al fútbol que se han perdido en las diferentes actividades de la vida cotidiana por falta de apoyo y muchas cosas más y no lograron llegar al fútbol profesional, son mucho los ejemplos tal es el caso de Ervin González un central con muy buenas condiciones, Cristian Franco un volante muy bueno, Hugo Valderrama un delantero etc. Asi por el estilo muchos más se fueron diluyendo hasta desaparecer completamente"

El jefe de programa de la Licenciatura en Educación Física, de la Universidad Surcolombiana Virgilio Puerto Polanco, afirma, "en todo el tiempo que estuve vinculado al fútbol huilense como entrenador de diferentes selecciones del departamento y del equipo profesional del Club Deportivo Atlético Huila en su momento, fueron muy pocos los jugadores que lograron ascender al equipo profesional del departamento y mucho menos al de otro lado" las razones indicadas por el docente en mención fueron muchas pero lo mas importantes es que al igual que los personajes anteriormente mencionados tiene la misma concepción acerca de que en el fútbol profesional colombiano el número de jugadores nacidos y formados en el departamento del Huila es muy escaso y deja en evidencia que este problema no es de ahora ya lleva tiempo y hasta el momento no se sabe de soluciones radicales al principal problema que está afectando al joven futbolista con aspiraciones de triunfar a nivel nacional e internacional.

En los últimos cuatro años de 84 jugadores en total que han terminado su etapa juvenil y que estarian en un estado óptimo y con las condiciones necesarias para conformar un equipo profesional tan solo 2 de ellos están inscritos en el fútbol profesional colombiano con el equipo profesional del Club Deportivo Atlético Huila, los otros 82 han ido desapareciendo paulatinamente del deporte en vista que no cuentan con la oportunidad de acceder al plantel profesional del departamento, y las posibilidades de ser enviado a otro equipo profesional en el fútbol colombiano son más escasas todavía.

Entonces la mayoría de futbolistas juveniles del departamento de Huila se ven en la difícil situación de desertar del deporte ya que este no brinda las oportunidades suficientes para lograr éxito y asegurar un futuro para él y su familia, y más bien obligan a que el deportista inicie otras actividades para el sustento propio y hasta en ocasiones que se desvié y tome el camino equivocado como la drogadicción, el alcoholismo y la delincuencia etc. 
Cuando no había fútbol profesional en nuestro departamento jugadores huilenses como Armando "La coneja" Acosta, tuvieron una larga trayectoria deportiva y conformaron selecciones Colombia para Juegos Olimpicos, creíamos que la solución estaba en que tuviéramos un equipo profesional para darle oportunidad a nuestros muchachos de acceder al profesionalismo, aún no se ha podido.

Hay que reconocer que no basta con entrenar para desarrollar un talento deportivo, es muy importante la competencia, y dado el reducido número tanto de equipos en categorias juveniles como de torneos y competencias desarrolladas por los organismos pertinentes en nuestro medio, el jugador local no tiene un escenario donde desarrollarlas ni quien admire y reconozca su talento para que les den esta oportunidad.

\section{Profesionales al servicio del Club}

En el área de rehabilitación, desde hace siete años está vinculado el Licenciado en Educación Física y especialista en rehabilitación deportiva Mauricio Murcia, quien ha demostrado idoneidad en el cargo y hoy en día se proyecta como uno de los mejores en su campo a nivel nacional. Como entrenador de divisiones se ha destacado el Licenciado Diego Armando Barreto Cortes al igual que Mauricio egresado de la Universidad Surcolombiana. Dada su experiencia e idoneidad fue llamado por un equipo profesional de Guatemala para asumir las funciones de preparador físico, sin embargo toda la experiencia la adquirió en el Atlético Huila y estos son dos ejemplos que pueden y deben multiplicarse a partir de más oportunidades a los profesionales huilenses.

\section{La dirigencia deportiva}

El mayor accionista del Club Atlético Huila es el industrial y comerciante Orlando Rojas Bustos, de origen huilense quien ha designado como presidente al Doctor Jorge Fernando Perdomo, también oriundo del departamento del Huila desarrollando una excelente labor desde el punto de vista administrativo y deportivo, al respecto el presidente afirma "pienso que si esta institución la ven el Gobierno departamental, patrocinadores, y huilenses, como una empresa de todos, seguramente podremos llegar mucho más lejos de lo que hemos logrado hasta ahora"

Sabemos de las limitaciones de diversa indole con las que la dirigencia del Club Atlético Huila tiene que lidiar frente a avezados dirigentes en el seno de la Dimayor para sacar adelante el equipo en el plano deportivo y administrativo, sin embargo es gratificante conocer de acuerdo a sus palabras "El prestigio del Atlético Huila en la Federación Colombiana de Fútbol y la Dimayor es muy alto, hay un gran respeto por la institución. Tanto asi que en muy breve tiempo he logrado acceder a tal vez la comisión más importante que tiene la Federación que es la presidencia de la comisión de planeación." Tenía razón el dirigente Jorge Fernando Perdomo, los resultados deportivos y el rigor administrativo mostrado en el manejo del Atlético Huila, lo llevaron a ocupar una silla en el comité ejecutivo de la Federación Colombiana de Fútbol.

¿Cómo podrían mejorar las oportunidades del Club hacia la comunidad?

Las principales motivaciones de los gobiernos departamental y municipal, para aportar recursos a esta Institución deportiva es tener un excelente canal de difusión de la región en contextos nacionales e internacionales y generar identidad y gratificación a la comunidad, sin embargo dado que no son despreciables estos aportes y no es excluyente con las motivaciones iníciales, el 
departamento del Huila y la ciudad de Neiva, deben exigir una cuota mínima de jugadores nativos de la región en el equipo, no con criterio chovinista, pero promover de manera más decidida al nuestro sobre todo cuando se sabe que tienen un similar nivel.

Recordemos que el "deporte para todos" es una función del Estado, no sólo con la intención de promover la recreación sino y especialmente para difundir la práctica de la actividad física como uno de los hábitos que determinan estilos de vida saludable, por lo tanto parte de estos recursos anualmente se deben invertir en la adquisición de un terreno donde funcione la Escuela de Formación Deportiva del Club y no en la cancha de Marte contigua al coliseo Álvaro Sánchez Silva, "tierrero" que no reúne las condiciones mínimas necesarias para adelantar prácticas con criterios pedagógicos..

Estas escuelas a pesar del poder de convocatoria que tienen, dado que se espera que formen la cantera del equipo profesional, son del Club Atlético Huila en asocio con los padres de familia quienes aportan una mensualidad para que sus hijos reciban la fundamentación deportiva requerida, recibe niños en edades desde los 5 años hasta los 18. Recordemos que el Contrato 214 de 2002 otorgado por la Secretaría de Hacienda al Club estipulaba $\$ 185.400 .000$.oo para el diseño, implementación y puesta en funcionamiento escuela de formación deportiva categorias edades 5 a 8 años, 9 a 12 años y 13 a 15 años destinados a personal técnico e idóneo. En la vigencia de 2003 la Secretaría de Hacienda Contrata con el Club la prestación de servicios para el fomento, fortalecimiento y desarrollo de escuelas de formación de talentos con miras a aportar más jugadores al equipo profesional que actúa en el campeonato Nacional, por un monto de $\$ 198.000 .000 .00$. Sin embargo durante estos 7 años de otorgado este contrato, todavía no ha actuado el primer jugador en el equipo profesional proveniente de estas escuelas.

En diciembre del 2008 el Club dio la noticia de que se había firmado una intención de convenio con uno de los mejores Clubes del mundo el Real Madrid de España, éste Club para dar el aval de la franquicia "sólo" exigía que la Escuela tuviera sus estándares de calidad. Por supuesto este exabrupto nunca se oficializó, tendriamos que destinar los recursos del Club para cumplir con dichos estándares. Una vez en funcionamiento esta utopía, sería excluyente con la gran masa de muchachos y familias ávidos de una oportunidad aunque sea en la escuela de campo Marte por módicos 30.000 pesitos mensuales.

Por el momento nos conformamos con ver a nuestro querido Club en la mitad de la tabla, aunque la verdad sea dicha en el lapso de tres años nos ha dado algunas satisfacciones que otros clubes de mayor arraigo desearian, como estas dos fallidas finales.

\section{Bibliografía}

Cantero Abad, L. (2007). La construcción de representaciones sociales a través del discurso textual. Estudios del hombre, 143 - 178.

Capistegui, F. (2001). Guerras danzadas. Fútbol e identidades locales y regionales en Europa. Pamplona: Universidad de Navarra.

Diego Armando, B. (Noviembre de 2008). El sinuoso recorrido del jugador de fútbol huilense al fútbol profesional. Neiva, Huila, Colombia.

Elias, N., \& Dunning, E. (1992). Deporte y ocio en el proceso de civilización. México: Fondo de Cultura Económica.

Macias Cervantes, C. F. (2006). Por una historia de las prácticas deportivas. Congreso Deporte Cultura y Sociedad, (pág. 2). Zacatecas.

Ordoñez, L. G. (13 de Diciembre de 2009). El rentado nacional. El Espectador, pág. 16.

Santacana Torres, C. (2006). El Barça y el Franquismo. Crónica de unos años decisivos (1968-1978). Madrid: Ediciones apóstrofe, S. L. 